\title{
Antipolar and Anticlinic Mesophase Order in Chromatin Induced by Nucleosome Polarity and Chirality Correlations
}

\author{
R. Garcés, ${ }^{1}$ R. Podgornik, ${ }^{2,3}$ and V. Lorman ${ }^{1}$ \\ ${ }^{1}$ Laboratoire Charles Coulomb, UMR 5221 CNRS-Université Montpellier 2, F-34095 Montpellier, France \\ ${ }^{2}$ Department of Theoretical Physics, J. Stefan Institute, SI-1000 Ljubljana, Slovenia \\ ${ }^{3}$ Department of Physics, Faculty of Mathematics and Physics, University of Ljubljana, 1000 Ljubljana, Slovenia
}

(Received 17 October 2014; published 9 June 2015)

\begin{abstract}
Contrary to the usual "rigid supermolecular assembly" paradigm of chromatin structure, we propose to analyze its eventual ordered state in terms of symmetry properties of individual nucleosomes that give rise to mesophase order parameters, like in many other soft-matter systems. Basing our approach on the Landau-de Gennes phenomenology, we describe the mesoscale order in chromatin by antipolar and anticlinic correlations of chiral individual nucleosomes. This approach leads to a unifying physical picture of a whole series of soft locally ordered states with different apparent structures, including the recently observed heteromorphic chromatin, stemming from the antipolar arrangement of nucleosomes complemented by their chiral twisting. Properties of these states under an external force field can reconcile apparently contradictory results of single-molecule experiments.
\end{abstract}

DOI: 10.1103/PhysRevLett.114.238102

PACS numbers: 87.16.Sr, 64.70.mf, 87.16.A-

Genomic DNA in eukariotic cells is highly compacted into a complex chromatin structure as a result of association with proteins. Chromatin ensures compaction and ordering during cell division, as well as a more swollen form for efficient transcription during interphase [1].

Chromatin organization on different scales has been widely studied over the last decades but the reliability of structural data and the underlying physical mechanisms still differs greatly from one level of organization to another. On the small scale, the basic structural unit was identified as the nucleosome core particle (NCP) [2]. This wedgecylinder-like particle is formed by a histone protein octameric core with 147 base pairs (bp) of DNA wrapped around it in a superhelical turn. Successive NCPs are joined by linker DNA, forming the "beads-on-a-string" $10 \mathrm{~nm}$ fiber structure. On the large scale, high-throughput molecular biology methods [3-5] report genome-wide distribution of contact probability between large-scale chromatin domains (about $\sim 10^{8}$ DNA bp) [6,7] not requiring any molecular-scale details [8].

On the intermediate mesoscale, comprising several thousand bp, the principles of architecture and the genome folding mechanism remain unclear. The ultimate biologically relevant goal, to bridge the small-scale protein complex action and the genome-wide-scale reaction, thus remains elusive. Typically for many soft condensed matter systems, e.g., liquid crystals [9] or polymers [10], it is not the exact geometry but rather the symmetry that defines their main characteristics [11], connecting the regimes of microscopic properties of rigid subunits and the fluctuation-dominated properties of the macroscopic aggregates [8,12]. Nevertheless, the main thrust of chromatin studies was directed solely toward establishing models of a "molecular" structure, focusing on the socalled 30-nm fiber with a rigid arrangement of nucleosomes $[13,14]$ : the solenoid $[15,16]$, the two-start helix $[17,18]$, and the crosslinker [19] models, or more physical models [20-22] based on linker backbone constraints.

To this paradigm we counterpose the ordered chromatin structure unlike a "rigid supermolecular assembly" but rather like an ordered mesophase [9] induced by shortrange correlations of individual $\mathrm{NCP}$ properties and described in terms of mesophase order parameters. This description of the mesoscale order is also consistent with the observed in vivo chromatin heterogeneity and softness of the structure [14]. In this perspective the mesoscale structure is also dependent on the low-energy fluctuational modes, on topological defects, and on the confinement $[9,23]$.

Group theoretical analysis within the Landau-de Gennes framework [9] allows us to describe the mesoscale order in chromatin by chiral antipolar and anticlinic correlations of NCP mesogenic properties. We define three mesophase order parameters (OPs): antipolar orientation of nucleosome dyad axes, antipolar shifting of neighboring nucleosomes, and anticlinic tilting of nucleosome superhelical axes with respect to the local axis of the structure. These OPs (i) have the same symmetry due to the chirality of the system, and thus, are proportional to one another; (ii) the helical winding of the structure is induced by chiral twisting of these OPs; (iii) a series of soft locally ordered states differing in apparent structures are induced by the same mechanism resulting in a short-scale cancellation of polar properties of chromatin. For the simplest case of a chiral antipolar and anticlinic structure its behavior under an external force field is characterized by two distinct 
threshold force values for highly ordered structure, while for weakly ordered structure the theory predicts a direct transition from the locally helical fiber state to the beadson-a-string state. These results can explain the differences in putative chromatin structures based on the singlemolecule experiments $[24,25]$. The approach developed here constitutes the basis for interpretation of recent advances in cryo-electron-microscopy (CEM) of chromatin in vivo as well as in vitro, showing heteromorphic structures [26-31].

Mesogenic properties of NCPs are characterized by their shape and charge anisotropy, stemming from a short (1.7 turns) DNA superhelical wrapping around the histone core [2], and resulting in their polarity and chirality. The DNA entry-exit point defines a polar dyad axis [Fig. 1(b) insert]. This transverse "polarization" determines the orientation of the NCPs visible also in CEM [Figs. 1(a) and (b)] [32]. The approach based on correlations of the mesogenic properties of DNA-histone complexes [33] has been instrumental in the study of condensed solutions of NCPs [32,34]. At sufficiently high osmotic pressure NCPs aggregate into columns with correlated polar dyad axes [Fig. 1(b)], while columns form arrays of different complexity as a function of monovalent salt concentration [35]. The mechanism which drives the ordering results in structures that are locally polar but globally antipolar, with their average polarization canceled out within the local "unit cell." Similar mesoscopic nucleosome-nucleosome interactions of antipolar type could be responsible also for the chromatin ordering, observed in similar physicochemical conditions (nucleosome density, ionic strength, etc.), and could be instrumental for selecting possible ordered chromatin structures.

Our model fiber is defined as a one-dimensional array of NCPs joined by DNA linkers and positioned along DNA at an average distance $d$. In the reference disordered state the mutual orientations of NCP dyad axes are random, their

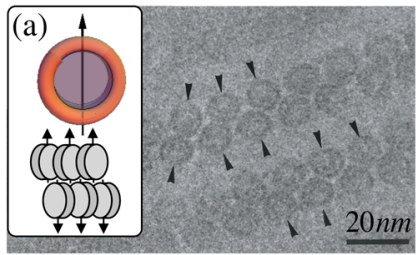

(c)

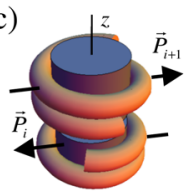

(d)
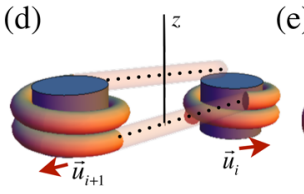

(e)

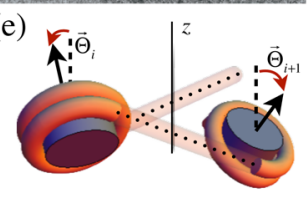

FIG. 1 (color online). Antipolar ordering. (a) Antipolar ordering of dyad axes in NCP columns (CEM of vitreous sections [32]). (b) Correlation of NCP dyad axes along the column. Inserts: NCP polar dyad axis location and schematic organization of NCP. Pseudoproper order parameters: (c) Antipolar orientation of dyad axes. (d) Antipolar shift. (e) Anticlinic tilt of superhelix axes. organization similar to a beads-on-a-string $10 \mathrm{~nm}$ fiber, obtained by decondensation of chromatin [14]. Orientations of the polar vectors $\mathbf{P}_{i}$ associated with the dyad axes of nucleosomes are also randomly distributed, preserving the local cylindrical symmetry of the array, while the fixed sign of chirality of individual NCPs (lefthanded) makes beads-on-a-string fiber globally chiral. The resulting point symmetry group of the disordered reference state is $D_{\infty}$. Its space group $D_{\infty} \wedge T_{d}$ is completed by a "translation" $T_{d}$ along the fiber. We are interested in ordering that results in antipolar dyad axes orientations, i.e., with $\mathbf{P}_{i}$ correlated, but with resultant "polarization" $\mathbf{P}=\sum_{i} \mathbf{P}_{i}$ canceled on a short "unit-cell" scale. Note that due to the chirality of the disordered state the ordering in this medium is qualitatively different with respect to the spontaneous chiral symmetry breaking in a packing of achiral subunits (e.g., Ref. [36]). Polar vector $\mathbf{P}_{i}$, displacement vector $\mathbf{u}_{i}$, and axial vector $\boldsymbol{\Theta}_{i}$ of the NCP superhelix axis tilt span the same irreducible representation of this group and thus have to be linearly dependent, proportional to one another in the simplest case (see Supplemental Material [37]). This allows us to limit strongly the number and the structures of possible ordered states.

Consider $\mathbf{P}_{i}$ 's oriented on average normal to the $z$ axis. Then transitions from the beads-on-a-string state to antipolar ordered states are driven by the condensation of a transverse polar wave,

$$
\begin{aligned}
\Pi(z)= & \left\{\rho_{1} \cos \left(k z+\phi_{1}\right)+\rho_{2} \cos \left(k z-\phi_{2}\right)\right\} \mathbf{e}_{x} \\
& +\left\{-\rho_{1} \sin \left(k z+\phi_{1}\right)+\rho_{2} \sin \left(k z-\phi_{2}\right)\right\} \mathbf{e}_{y},
\end{aligned}
$$

where $\mathbf{e}_{x}$ and $\mathbf{e}_{y}$ are the unit vectors in the plane perpendicular to the $z$ axis, $\rho_{1}, \rho_{2}, \phi_{1}$ and $\phi_{2}$ are the wave amplitudes and phases, and $k$ is the wave vector. The average values of $\mathbf{P}_{i}$ for individual NCPs are obtained by inserting their coordinates into Eq. (1). The ensemble of ordered states driven by Eq. (1) is described in the Landau theory frame [38] based on group theoretical analysis.

Group theory $[38,39]$ distinguishes three qualitatively different classes of ordered states driven by $\Pi(z)$, depending on the number $N$ of neighboring NCPs involved in cancellation of polarization P: (i) $k=0$, with parallel ordering of NCP dyad axes (locally not antipolar); (ii) $k= \pm \pi / d$, with antipolar ordering involving two neighboring NCPs $(N=2)$ and with only one ordered state in which dyad axes of neighboring NCPs are simply antiparallel and form a local "zigzag"; (iii) $-\pi / d<k<\pi / d$, with antipolar wave involving more than two NCPs $(N>2)$ in the local "unit cell." This class contains an infinite number of possible waves with the wave vectors $k=2 \pi / N d$. In addition, for each fixed value of $N>2$ the wave results in five possible ordered states (see below).

In the simplest antipolar case $N=2$, the wave Eq. (1) is reduced to the antiparallel orientation of neighboring NCPs 
[Fig. 1(c)]. The OP which drives the transition to this ordered state is the "antipolarization" vector $\mathbf{A}_{p}=\mathbf{P}_{i}-\mathbf{P}_{i+1}$. The vector of antiparallel displacement [Fig. 1(d)] of neighboring NCPs $\mathbf{A}_{u}=\mathbf{u}_{i}-\mathbf{u}_{i+1}$ is directly proportional to $\mathbf{A}_{p}$ and together with the linker DNA elasticity determines the "center-to-center" distance between them. The axial vector $\mathbf{A}_{\Theta}=\boldsymbol{\Theta}_{i}-\boldsymbol{\Theta}_{i+1}$ of anticlinic tilt of superhelix axes of neighboring NCPs [Fig. 1(e)] is also proportional to $\mathbf{A}_{p}$. These three linearly dependent OP (pseudoproper OP [40]) determine the local "zigzag" structure, systematically observed in chromatin at low ionic strengths $[14,30]$ and the "double tracks" of NCPs observed at higher salt concentrations [30]. Antiparallel orientation of neighboring NCPs accompanied by their anticlinic tilt is evident also in the crystal structure of tetranucleosome [41,42]. Exactly the same local structure was observed in condensed NCP solutions [Fig. 1(a)] [32], with anticlinic tilt in neighboring columns [34] even in the absence of the linker DNA.

The Landau-de Gennes free energy of this transition can be expressed in terms of $\mathbf{A}_{p}$ only, two other pseudoproper OPs, $\mathbf{A}_{u}$ and $\mathbf{A}_{\Theta}$, being proportional to $\mathbf{A}_{p}$. Taking the twocomponent $\mathrm{OP}$ in the form $\tilde{A}_{p}=A_{p} e^{i \phi}$, we obtain the minimal model of the free energy density:

$$
F_{N=2}=\frac{a}{2} A_{p}^{2}+\frac{b}{4} A_{p}^{4}-\Lambda A_{p}^{2}\left(\frac{d \phi}{d z}\right)+\frac{K}{2} A_{p}^{2}\left(\frac{d \phi}{d z}\right)^{2}
$$

Here the first two terms represent the energy of homogeneous ordering, independent of the orientation (given by $\phi$ ) of the ordered "zigzag" structure with respect to the laboratory frame. However, the chiral symmetry of the disordered beads-on-a-string state enforces a third term due to chiral torsion, linear in the spatial derivative of $\phi . \Lambda$ is the mesoscopic chiral strength of the medium, dependent on the NCP density and the ionic strength. The last term is due to the usual energy of torsional rigidity. The Euler-Lagrange equations lead to the equilibrium solution: $\phi(z)=q_{0} z+\phi_{0}$. From the definition of $\mathbf{A}_{p}$ we obtain the distribution of the NCP dyad axes in the form of a two-start helix with the wave vector $q_{0}=\Lambda / K$ and the pitch of the helix given by $p=2 \pi / q_{0}=2 \pi(K / \Lambda)$ [Figs. 2(a)-2(d)]. Note that variation of the mesophase helix pitch $p$ [cf. Figs. 2(b) and 2(c)] or change of the tilt sign [cf. Figs. 2(c) and 2(d)] as a function of external conditions (i.e., ionic strength) result in different apparent structures, though the theory proposed shows that they belong to the same mesophase. This result would be quite difficult to justify in a rigid supermolecular assembly approximation.

For antipolar orderings involving $N>2$ nucleosomes, the homogeneous part of the free energy density $F_{N}^{0}$ depends on two amplitudes $\rho_{1}$ and $\rho_{2}$ of the wave $\Pi(z)$ and on the phase difference $\delta=\phi_{1}-\phi_{2}$. The inhomogeneous part of the free energy depends on derivatives of the sum $\phi_{1}+\phi_{2}$ with respect to the coordinate $z$ and makes the OP distribution helical. Minimization of $F_{N}^{0}$ for a fixed $N>2$ gives rise to five ordered states (see Supplemental Material [37] for details): (I) $\rho_{1} \neq 0, \rho_{2}=0$; (II) $\rho_{1}=0$, $\rho_{2} \neq 0$; (III) $\rho_{1} \neq \rho_{2} \neq 0, \cos (m \delta)=1$; (IV) $\rho_{1} \neq \rho_{2} \neq 0$, $\cos (m \delta)=-1$, and $(\mathrm{V}) \rho_{1} \neq \rho_{2} \neq 0, \cos (m \delta) \neq \pm 1$. Here $m=N$ for odd $N$ and $m=N / 2$ for even $N$. States I and II are simple generalizations of the "zigzag" structure. The "unit cell," resembling a succession of rotated and tilted zigzags, contains $N$ NCPs with the dyad axes orientations successively rotated by $\Delta \varphi_{i, i+1}=2 \pi / N$ and shifted along the $z$ axis. The same holds for orientations of the axial tilt vectors $\boldsymbol{\Theta}_{i}$ with $\left|\boldsymbol{\Theta}_{i}\right|=\Theta$. However, states I and II with $N>2$ are qualitatively different with respect to simple zigzag state and can be obtained from it by way of phase transition only. A stack of such unit cells for $N=3$ in the state II is presented in Fig. 2(e). Phases III-V are more complex. Their symmetry is locally biaxial, with inhomogeneous distribution of, either azimuthal angles $\Delta \varphi_{i, i+1}$, or of the tilt values $\Theta_{i}$ (see Supplemental Material [37], Figs. 1 and 2). For example for $N=3$ in phase III the azimuthal angles of the dyad axes' directions are distributed inhomogeneously, the angles between the directions of three axes are $\Delta \varphi_{12}=\Delta \varphi_{31}>\Delta \varphi_{23}$, and in contrast to uniaxial states I and II the tilts of nucleosomes in state III are no more equal $\Theta_{2}=\Theta_{3}<\Theta_{1}$ [see Fig. 2(g)]. (a)

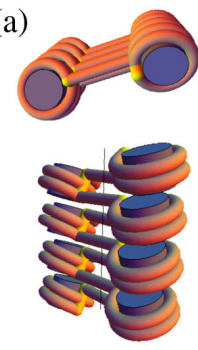

(b)

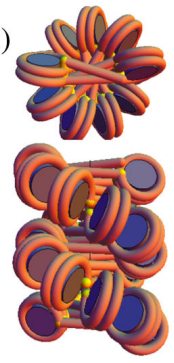

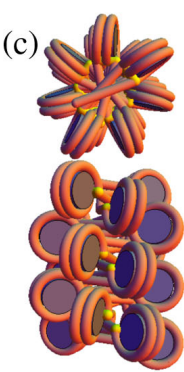

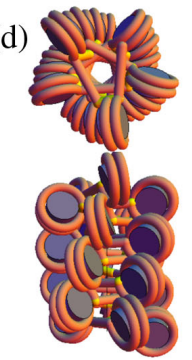

(e)

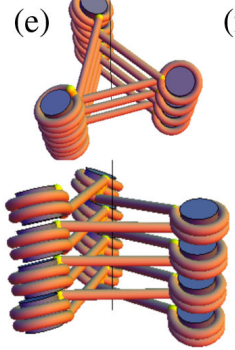

(f)

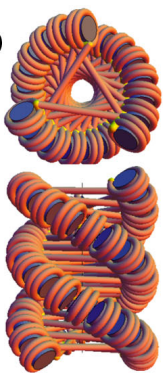

(g)

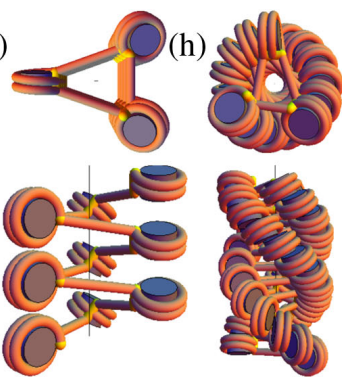

FIG. 2 (color online). Calculated locally helical structures induced by the chiral twisting of the antipolar order (top and side views). Structures with $N=2$ (a)-(d) and $N=3$ (e)-(h) NCPs involved in polarization cancellation. (a), (e), and (g): Structures with vanishing chiral strength $\Lambda \rightarrow 0$. (b), (c), and (f): Structures with $\Lambda \neq 0$ and positive tilt $\boldsymbol{\Theta}_{i} ; \Lambda_{b}<\Lambda_{c}$. (d): Structure with negative tilt $\boldsymbol{\Theta}_{i} ; \Lambda_{d}=\Lambda_{c}$, (g),(h): Biaxial phase III structures. 
Understanding the effects of chiral twisting for $N>2$ requires the minimization of the inhomogeneous free energy $F_{N}$ which depends on $\phi_{1}, \phi_{2}$ and their derivatives,

$$
F_{N}=F_{N}^{0}-\sum_{i=1,2}\left[\Lambda_{i} \rho_{i}^{2}\left(\frac{d \phi_{i}}{d z}\right)-\frac{K_{i}}{2} \rho_{i}^{2}\left(\frac{d \phi_{i}}{d z}\right)^{2}\right] .
$$

The Euler-Lagrange equations lead to the system of two differential equations of the $N$ sine-Gordon type and, consequently, to a complex dependence of angles $\phi_{1}$ and $\phi_{2}$ on $z$. But for the simplest state I (or II) the OP amplitude $\rho_{1}$ (or $\rho_{2}$ ) vanishes. The problem is then reduced to Eq. (2) by simply replacing $A$ with $\rho_{2}$ and $\phi$ by $\phi_{2}$ leading to a $N$-start helix with wave vector $q$ and pitch $p$, independent of $N$. In biaxial states III-V helices become biaxial also. Azimuthal distances between strands become different as well as the nucleosome tilts in different strands [see Fig. 2(h)]. In the near future the search for local fiber biaxiality can constitute an interesting direction in the studies of chromatin architecture.

In nuclei of living cells the chromatin fiber is constantly submitted to flows and to the mechanical action of protein complexes inducing external forces and torques (for setups approaching this situation in vitro see Ref. [43]). The energy of the chiral helical twisting is weak compared to the antipolar ordering and especially compared to the energy of DNA-protein complexation. Thus, rather weak external fields can induce distortion from the helicoidal structure to more biologically relevant inhomogeneous or unwound states. This property makes the chromatin fiber dynamic and facilitates mechanisms assisting genetic information processing.

To describe the behavior of the model chromatin fiber under external force fields, we choose the simplest twostart helix structure $(N=2)$ with free energy Eq. (2) together with the coupling terms with the global external force field $\mathbf{F}$, assumed to be normal to the $z$ axis,

$$
F_{\text {field }}=F_{N=2}+\gamma_{1} A_{p}^{2} P^{2}+\gamma_{2}\left(\mathbf{A}_{p} \cdot \mathbf{P}\right)^{2}+\frac{\alpha}{2} P^{2}-(\mathbf{F} \cdot \mathbf{P}) .
$$

Here we used proportionality between displacement vector $\mathbf{u}$ and "polarization" $\mathbf{P}$, as well as between antiparallel vectors $\mathbf{A}_{u}$ and $\mathbf{A}_{p}$. For sufficiently strong antipolar ordering the behavior of the helical structure under this force field is characterized by two threshold force values describing: (i) helix unwinding into a homogeneous zigzag structure; (ii) transition from the antiparallel zigzag to the parallel beads-on-a-string state.

The minimization of free energy, Eq. (4), leads to solutions of an integral equation (see Supplemental Material [37]) which describe complex inhomogeneous states. These solutions exist only in the range $0<F<F_{c}$, where $F_{c}$ is given by $F_{c}=(\pi / 2) q_{0} \sqrt{K A_{p}^{2}\left(2 \gamma_{1} A_{p}^{2}+\alpha\right)}$ with $q_{0}$ and $A_{p}$ being the equilibrium values of the helix wave vector and antipolar OP, respectively. The complete phase diagram of the force field induced behavior is shown in Fig. 3. Three states: helicoidal, homogeneous antiparallel ('zigzag), and homogeneous parallel (beads-on-a-string) coexist at the Lifshitz point (LP) (see, e.g., Ref. [11]) with coordinates $a_{\mathrm{LP}}=-\Lambda^{2} \gamma_{1} / K \gamma_{2}, \quad F_{\mathrm{LP}}=(\Lambda \alpha / K) \sqrt{K / \gamma_{2}}$, separating two possible thermodynamic scenarios (dotted arrows in Fig. 3) for the transition from the helical to the beads-on-a-string structure. Below the LP this transition is akin to a continuous "phase transition" induced by a rather weak force. Beyond the LP the transition takes place in two steps described above, with the intermediate zigzag structure stable for $F>F_{c}$. The transition from the zigzag to the beads-on-a-string state changes its order at the tricritical point (TCP) with the coordinates $a_{\mathrm{TCP}}=-b \alpha / 4 \gamma_{1}, F_{\mathrm{TCP}}=\sqrt{b \alpha^{3} / 8 \gamma_{1}^{2}}$.

Though the constraint-free helical structure is taken here to be a two-start helix, its behavior along the first thermodynamic path is characterized by only one threshold force. Such a behavior in single-molecule experiments is usually interpreted as a signature of the one-start solenoid structure. The calculated phase diagram (Fig. 3) shows that this behavior can also be a signature of the weak order in the two-start helix structure. It can then explain apparent differences in single-molecule experiments of several groups $[24,25]$.

The order in mesophases is usually much weaker than in more correlated systems [9] and is subject to strong fluctuations. It makes the correlation of dyad axes' directions short range and diffuse, enabling formation of topological defects. The simplest defects of the antipolar order are locally parallel polar arrangements of neighboring

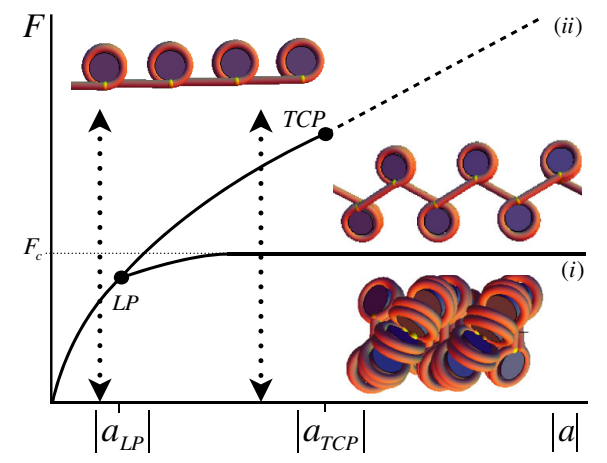

FIG. 3 (color online). Phase diagram of the force-induced transitions. Thermodynamic paths are shown by dotted arrows. Line (i) represents the transitions from the helical state (HS) to the zigzag state (ZS). In the Lifshitz point LP line (i) merges with line (ii) which describes direct continuous transitions between the HS and the beads-on-a-string state (BS) for $|a|<\left|a_{\mathrm{LP}}\right|$. For $\left|a_{\mathrm{LP}}\right|<$ $|a|<\left|a_{\mathrm{TCP}}\right|$ the second-order transitions between the ZS and BS take place on this line, while for $|a|>\left|a_{\mathrm{TCP}}\right|$ the ZS-to-BS transitions are first order. 
nucleosomes. The resulting structure is "heteromorphic," consisting of regions with short-range antiparallel helical order connected by very narrow defect regions with parallel arrangement (see Supplemental Material [37], Fig. 3). Such structures were seen experimentally in a range of conditions [29-31]. For orderings with $N>2$ (especially for biaxial states III-V), additional degrees of freedom promote complex "interdigitated" structures frequently observed in chromatin $[14,26]$. Mesophase order, low-energy excitation modes, and easy defect formation evidenced by the theory constitute the basis for the understanding of chromatin interaction with ATP-dependent protein complexes involved in transcription and cell division.

V. L. acknowledges financial support from the Laboratory of Excellence NUMEV. R.P. acknowledges Slovenian Research Agency (ARRS) Grant No. P1-0055. R. G. acknowledges financial support from Becas Chile program.

[1] B. Alberts et al., Molecular Biology of the Cell, 5th ed. (Garland Science, New York, 2008).

[2] K. Luger, K. Luger, A. W. Mäder, R. K. Richmond, D. F. Sargent, and T. J. Richmond, Nature (London) 389, 251 (1997).

[3] J. Dekker, K. Rippe, M. Dekker, and N. Kleckner, Science 295, 1306 (2002).

[4] J. Dekker, Nat. Methods 3, 17 (2006).

[5] J. Dekker, M. A. Marti-Renom, and L. A. Mirny, Nat. Rev. Genet. 14, 390 (2013).

[6] T. B. K. Le, M. V. Imakaev, L. A. Mirny, and M. T. Laub, Science 342, 731 (2013).

[7] G. Cavalli and T. Misteli, Nat. Struct. Mol. Biol. 20, 290 (2013).

[8] J. D. Halverson, J. Smrek, K. Kremer, and A. Y. Grosberg, Rep. Prog. Phys. 77, 022601 (2014).

[9] P. G. de Gennes and J. Prost, The Physics of Liquid Crystals (Claredon Press, Oxford, 1993).

[10] P. G. de Gennes, Scaling Concepts in Polymer Physics (Cornell University Press, 1979).

[11] P. M. Chaikin and T. C. Lubensky, Principles of Condensed Matter Physics (Cambridge University Press, Ithaca, 2000).

[12] S. Meyer, N. B. Becker, S. H. Syed, D. Goutte-Gattat, M. S. Shukla, J. J. Hayes, D. Angelov, J. Bednar, S. Dimitrov, and R. Everaers, Nucleic Acids Res. 39, 9139 (2011).

[13] K. van Holde and J. Zlatanova, Semin. Cell Dev. Biol. 18, 651 (2007).

[14] S. A. Grigoryev and C. L. Woodcock, Exp. Cell Res. 318, 1448 (2012).

[15] J. T. Finch and A. Klug, Proc. Natl. Acad. Sci. U.S.A. 73, 1897 (1976).

[16] J. Widom and A. Klug, Cell 43, 207 (1985).

[17] R. A. Horowitz, D. A. Agard, J. W. Sedat, and C. L. Woodcock, J. Cell Biol. 125, 1 (1994).

[18] C. L. Woodcock, L. L. Y. Frado, and J. B. Rattner, J. Cell Biol. 99, 42 (1984).
[19] S. P. Williams and J. P. Langmore, Biophys. J. 59, 606 (1991).

[20] H. Schiessel, W. M. Gelbart, and R. Bruinsma, Biophys. J. 80, 1940 (2001).

[21] M. Depken and H. Schiessel, Biophys. J. 96, 777 (2009).

[22] H. Boroudjerdi, A. Naji, and R. Netz, Eur. Phys. J. E 34, 72 (2011).

[23] M. Kleman and O. D. Lavrentovich, Soft Matter Physics: An Introduction (Springer, New York, 2003).

[24] Y. Cui and C. Bustamante, Proc. Natl. Acad. Sci. U.S.A. 97, 127 (2000).

[25] M. Kruithof, F.-T. Chien, A. Routh, C. Logie, D. Rhodes, and J. van Noort, Nat. Struct. Mol. Biol. 16, 534 (2009).

[26] C. L. Woodcock and P. R. Ghosh, Cold Spring Harbor Persp. Biol. 2, a000596 (2010).

[27] M. Eltsov, K. M. MacLellan, K. Maeshima, A. S. Frangakis, and J. Dubochet, Proc. Natl. Acad. Sci. U.S.A. 105, 19732 (2008).

[28] K. Maeshima, S. Hihara, and M. Eltsov, Curr. Opin. Cell Biol. 22, 291 (2010).

[29] M. P. Scheffer, M. Eltsov, and A. S. Frangakis, Proc. Natl. Acad. Sci. U.S.A. 108, 16992 (2011).

[30] M. P. Scheffer, M. Eltsov, J. Bednar, and A. S. Frangakis, J. Struct. Biol. 178, 207 (2012).

[31] S. A. Grigoryev, G. Arya, S. Correll, C. L. Woodcock, and T. Schlick, Proc. Natl. Acad. Sci. U.S.A. 106, 13317 (2009).

[32] A. Leforestier, J. Dubochet, and F. Livolant, Biophys. J. 81, 2414 (2001).

[33] V. Lorman, R. Podgornik, and B. Žekš, Europhys. Lett. 69, 1017 (2005).

[34] F. Livolant, S. Mangenot, A. Leforestier, A. Bertin, M. de Frutos, E. Raspaud, and D. Durand, Phil. Trans. R. Soc. A 364, 2615 (2006).

[35] S. Mangenot, A. Leforestier, D. Durand, and F. Livolant, Biophys. J. 84, 2570 (2003).

[36] G. T. Pickett, M. Gross, and H. Okuyama, Phys. Rev. Lett. 85, 3652 (2000).

[37] See Supplemental Material at http://link.aps.org/ supplemental/10.1103/PhysRevLett.114.238102 for the discussion of pseudo-proper order parameters and classification and illustration of some ordered, inhomogeneous and heteromorphic chromatin states.

[38] L. D. Landau and E. M. Lifshitz, Statistical Physics, 3rd ed. (Pergamon Press, Oxford, 1980).

[39] J. P. Elliot and P. G. Dawber, Symmetry in Physics (Oxford University Press, New York, 1979).

[40] J. C. Toledano and P. Toledano, The Landau Theory of Phase Transitions (World Scientific, Singapore, 1987).

[41] B. Dorigo, T. Schalch, A. Kulangara, S. Duda, R. R. Schroeder, and T. J. Richmond, Science 306, 1571 (2004).

[42] T. Schalch, S. Duda, D. F. Sargent, and T. J. Richmond, Nature (London) 436, 138 (2005).

[43] C. Lavelle, J. M. Victor, and J. Zlatanova, Int. J. Mol. Sci. 11, 1557 (2010). 